\title{
Letter regarding article by Hyun Jung Kim et al.: Total ankle arthroplasty versus ankle arthrodesis for the treatment of end-stage ankle arthritis: a meta-analysis of comparative studies
}

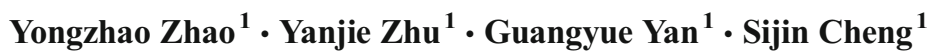

Received: 12 October 2016 / Accepted: 21 October 2016 / Published online: 29 October 2016

(C) SICOT aisbl 2016

\section{Dear Editor:}

In International Orthopaedics, Hyun Jung Kim et al. conduct a meta-analysis titled "Total ankle arthroplasty versus ankle arthrodesis for the treatment of end-stage ankle arthritis: a metaanalysis of comparative studies"; who have done a good job [1]. However, we have a few concerns about the methods and conclusions of this meta-analysis and are interested in the authors' response:

1. Though the authors declared that ten studies were included in the final meta-analysis, the analyzed studies of several items was much less than ten, such as post-operative VAS, complications, re-operations, and so on. Thus the conclusion of each item should be interpreted with caution.

2. The publication history indicated that International Orthopaedics received this meta-analysis on June 7th, 2016. However, the authors only searched the MEDLINE, EMBASE, and Cochrane library databases from January 1973 through May 2015. The principle quality of a good meta-analysis should be containing all the latest and relevant studies. In our opinion, the authors definitely should update the literature search before submitting to this journal.

3. In the statistical analysis, though the authors declared that random-effect model should be used if significant hetero-

Yongzhao Zhao

yongzhao_zhao@163.com

1 Tongji University School of Medicine, Shanghai, China geneity was observed, they did not report the exact standard to use this model in the forms of $\mathrm{I}^{2}$ values. Therefore, the model in this study is quite confusing.

4. The post-operative SF-36 (MCS), re-operations, and patient satisfaction showed no significant heterogeneity, however the authors still applied the random-effect model rather than fixed-effect model to analyze them. This was not consistent with the part of methods, which the authors should explain.

5. The authors found there was no significant difference of post-operative AOFAS score between total ankle arthroplasty (TAA) group and ankle arthrodesis (AA) group. However, there is an obvious heterogeneity. We did a recalculation of this data and found that heterogeneity could be distinctly reduced when Esparragoza 2011 study was excluded $\left(\mathrm{I}^{2}=81 \%\right.$ to $\left.0 \%\right)$. Therefore, the authors should look for the source of heterogeneity and include it in discussion.

The authors have made a great contribution in comparing the safety and efficacy of total ankle arthroplasty with ankle arthrodesis based on the available studies. However, further prospective studies in larger populations with long-term follow-up are still needed to draw a conclusion.

\section{Reference}

1. Kim HJ, Suh DH, Yang JH, Lee JW, Kim HJ, Ahn HS, Han SW, Choi GW (2016) Total ankle arthroplasty versus ankle arthrodesis for the treatment of end-stage ankle arthritis: a meta-analysis of comparative studies. Int Orthop 1-9. doi:10.1007/s00264-016-3303-3 